\title{
Piperazine Citrate
}

National Cancer Institute

\section{Source}

National Cancer Institute. Piperazine Citrate. NCI Thesaurus. Code C47674.

The citrate salt of piperazine, a cyclic amine with anthelminthic property. Piperazine citrate is a gamma-aminobutyric acid GABA receptor agonist and blocks the activity of acetylcholine at the neuromuscular junction thereby altering neuronal cell membrane permeability and causing hyperpolarization. This results in flaccid paralysis of worm musculature allowing the worm to become dislodged by peristalsis and expelled from the body. 